\title{
Anterior cervical interbody fusion with hydroxyapatite graft and plate system
}

\author{
Michael Bruneau, M.D., Jean-Francois Nisolle, M.D., Claude Gilliard, M.D., \\ AND THIERRY GuSTIN, M.D. \\ Departments of Neurosurgery and Radiology, University Hospital of Mont-Godinne, Université \\ Catholique de Louvain, Yvoir, Belgium
}

\begin{abstract}
Object. In cases of spondylosis or spine trauma, cervical interbody grafts are sometimes required after anterior discectomy. To avoid morbidity related to the harvesting of iliac crest bone, numerous materials have been developed such as allografts, methylmethacrylate, biocompatible osteoconductive polymer, and coralline grafts or cages. Some of these materials, however, are inefficient for fusion or are associated with specific complications. Conversely, hydroxyapatite (HA) grafts have numerous advantages. For example, their mechanical properties provide adequate load resistance and their porosity allows infiltration by newly formed bone, leading to complete fusion. The authors studied the results of using HA grafts combined with plating in patients who underwent anterior cervical discectomy.

Methods. Fifty-four patients underwent 68 cervical interbody fusion procedures in which an HA graft and plating were used. Indications for surgery were radiculopathy (caused by soft-disc herniation or spondylosis) in 46 cases, spondylotic myelopathy in two cases, and spinal trauma in six cases. Postoperatively the patients were followed for a mean of 24.6 months. Patients underwent radiography to evaluate fusion, intervertebral disc height, and the degree of lordosis.

Clinically, excellent or good results (based on the Odon classification) were demonstrated in $91 \%$ of patients who presented with radiculopathy. Complete interbody fusion was achieved in $99 \%$ of all cases. Preoperative kyphotic deformities were corrected in all cases after surgery. Intervertebral disc height was maintained throughout follow up. Surgery-related morbidity was low; only one patient suffered from a permanent dysphagia. Graft deterioration was observed in 13 cases and two graft fractures occurred but without adversely affecting fusion. There were no cases of graft extrusion.

Conclusions. Hydroxyapatite grafts are very efficient in achieving cervical fusion, maintaining intervertebral disc height, and restoring lordosis. When combined with the placement of a cervical plate, immediate stability is achieved and graft displacement is prevented.
\end{abstract}

KEY WORDS - hydroxyapatite graft • plate • spinal fusion • cervical spine discectomy

Since its first description in the early $1950 \mathrm{~s},{ }^{8,10,39}$ the anterior approach to the cervical spine has gained wide acceptance for the treatment of degenerative diseases or fractures. Although simple discectomy is considered by some authors to be sufficient for treating soft-disc herniation, ${ }^{15,22,25,27,29,32,34-36}$ interbody fusion is required in other cases in which spondylotic myelopathy or trauma-induced instability is present. The quality of fusion, which, theoretically, will influence clinical outcome, depends mainly on the properties of the interbody graft and the graft placement technique. The choice of the graft material is therefore very important. In addition to promoting fusion, the graft should also allow correction of the kyphotic deformities ${ }^{2}$ and prevent intervertebral disc space narrowing ${ }^{31}$ which if not halted, can lead to the recurrence of radiculopathy or myelopathy.

Autologous bone has been shown to induce a solid fusion. It is, however, the source of frequent complications, particularly pain, related to its harvesting at the iliac

Abbreviations used in this paper: $\mathrm{HA}=$ hydroxyapatite; $\mathrm{MR}=$ magnetic resonance. crest. ${ }^{24,40}$ Although these complications are avoided when allografts are substituted for autologous bone grafts, the former are associated with a lower rate of fusion and a higher risk of collapse. ${ }^{1,6,45}$ Coralline grafts ${ }^{2}$ and most synthetic materials ${ }^{18,26,30,41}$ have been found by many authors to be inadequate for fusion. Recently, a $100 \%$ fusion rate was obtained after application of interbody cages..$^{5,16,28}$ In most cases, however, the cages were filled with autologous bone.

Hydroxyapatite grafts have the advantages of osteoconductive properties, ${ }^{11}$ good resistance to collapse, simplicity of use, and a physiological shape. The aim of this study was to evaluate the efficacy of HA grafts in terms of fusion, restoration of lordosis, and in maintenance of intervertebral disc height. The role of anterior plating in combination with the HA graft is also discussed.

\section{CLINICAL MATERIAL AND METHODS}

\section{Patient Population}

Between January 1995 and July 1999, 60 consecutive patients, aged 28 to 73 years, underwent anterior cervical 
TABLE 1

Patient sex, indications for surgery, and fusion levels

\begin{tabular}{lc}
\hline \hline Characteristic & No. of Patients \\
\hline no. of patients & 54 \\
sex & 22 \\
male & 32 \\
female & \\
indication for op & 46 \\
radiculopathy & 2 \\
myelopathy & 6 \\
trauma & \\
operated level & 1 \\
C3-4 & 1 \\
C4-5 & 15 \\
C5-6 & 23 \\
C6-7 & 14 \\
C5-6 \& C6-7 &
\end{tabular}

discectomy and interbody fusion in which an HA graft and plate system were used. Six patients lost to follow up were excluded from the study, including two patients who died of unrelated causes at 8 weeks and 6 months postoperatively, respectively. Of the 54 remaining patients, 22 were men and 32 were women (Table 1 ).

In 46 patients, the indication for surgery was radiculopathy (resistant to conservative treatment) caused either by a soft-disc herniation (28 cases) or spondylosis (18 cases). Two patients suffered from a spondylotic myelopathy, and six patients were treated for a posttraumatic cervical spine fracture or ligamentous instability.

A one-level fusion was performed in 40 patients (23 at C6-7, 15 at C5-6, one at C4-5, and one at C3-4) and a two-level fusion was performed in 14 patients (all at C5-6 and C6-7). Thus, 68 levels were studied.

\section{Surgical Procedure}

Surgery was performed via a standard right-sided anterior cervical approach. After incision of the anterior longitudinal ligament, the disc was completely removed and subchondral cartilage was carefully resected using curettes, without any drilling, for exposure of the cortical bone.

Spondylotic spurs and herniated disc fragments were removed in standard fashion to decompress the nerve roots and the spinal cord widely. The posterior longitudinal ligament was opened only in cases of extruded disc herniation.

Hydroxyapatite grafts (Synatite, SBM, France), made of pure synthetic calcium HA, are available in various heights and widths. They are trapezoid shaped to provide some degree of lordosis. Their superior and inferior surfaces are convex to fit the natural concavity of the vertebral endplates (Fig. 1). The size of the graft was chosen to attain a slight distraction of the disc space and was determined by insertion of a metallic phantom (Fig. 1). After placement of a Caspar intervertebral distractor, the HA graft was introduced in the disc space and positioned to ensure maximal contact with the cortical endplates.

In all cases, the procedure was completed by applying anterior plates. In the first 11 patients, fusion was performed using a Senegas or Orosco plate. In the other 43

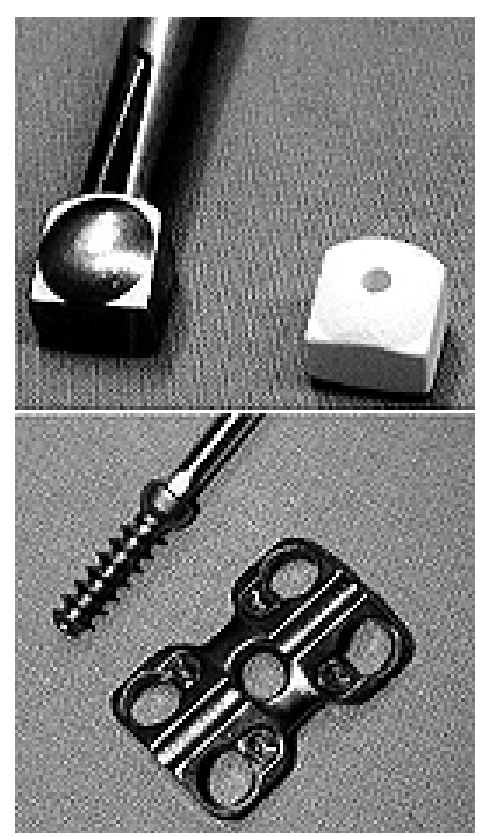

Fig. 1. Upper: Photograph showing the HA and metal phantom. Lower: Photograph showing the Codman plate with screw locking system.

patients, a Codman plate with a screw locking device (Johnson and Johnson, Raynham, MA) was used. Postoperatively, a soft cervical collar was applied for 6 weeks.

\section{Follow-Up and Radiological Studies}

Preoperative assessment included plain radiography, MR imaging, electromyography, and, in some cases, computerized tomography scanning. All patients underwent postoperative radiography to evaluate fusion, lordosis, and intervertebral disc height and to assess for graft-related complications. Plain radiography was performed at 3 days, 6 weeks, 3 to 6 months, 1 year, and then yearly until fusion was complete. The mean follow-up time was 24.6 months (range 7.4-58.2 months). The degree of lordosis was defined by the angle between two lines drawn parallel to the posterior wall of two adjacent vertebral bodies. Lordosis was systematically analyzed in the early postoperative period, and the condition of the spine was compared with preoperative status measured in eight patients in whom preoperative x-ray films were available. Postoperative MR imaging was performed in two patients.

Clinical outcome was assessed according to the Odon classification: an excellent or good result referred to complete or partial relief of symptoms with full return to activity; a fair result denoted improvement with some persistent limitation of activity; and a poor result indicated either no improvement or deterioration after surgery.

\section{RESULTS}

\section{Clinical Course}

At the last examination of the 46 patients with radiculopathy, 29 (63\%) were shown to have excellent postoperative outcomes and 13 (28\%) good outcomes, according 
TABLE 2

Summary of results and postoperative findings

\begin{tabular}{cc}
\hline \hline Finding & No. of Patients (\%) \\
\hline clinical result* & \\
excellent & 29 of $46(63)$ \\
good & 13 of $46(28)$ \\
fair & 4 of $46(9)$ \\
poor & 0 of $46(0)$ \\
fusion rate $\dagger$ & \\
1-level discectomy & 39 of $40(98)$ \\
2-level discectomy & 28 of $28(100)$ \\
total no. & 67 of $68(99)$ \\
pseudarthrosis $\dagger$ & 0 of $68(0)$ \\
postop lordosis & 54 of $54(100)$ \\
graft status $\dagger$ & \\
collapse & 2 of $68 \ddagger(3)$ \\
extrusion & 0 of $68(0)$ \\
fracture & 2 of $68 \S(3)$ \\
deterioration & 13 of $68 \S(19)$ \\
\hline
\end{tabular}

* According to the Odon classification system.

$\dagger$ Based on the total number of fused levels (68), not patients (54).

$\ddagger$ Less than $2 \mathrm{~mm}$.

$\S$ As determined radiologically.

to the Odon classification (Table 2). Four patients (9\%) in whom excellent or good results were demonstrated at 6 months were considered to have a fair result at the end of the study. Two of these patients complained of disabling cervical pain; this was probably explained by major degenerative changes in one case. The two other patients suffered residual radicular pain associated, in one case, with scapulohumeral periarthritis. Of the two cases with myelopathy, the symptoms stabilized in one and worsened in the other. In the posttraumatic group, five patients without preoperative deficits were asymptomatic after surgery and one remained quadriplegic.

On the whole, 19 patients experienced mild or moderate postoperative cervical pain. Of these, however, multilevel degenerative changes had been demonstrated preoperatively in 13 patients. The only surgery-related complica- tion was one case of severe, permanent dysphagia, probably due to prominence of the plate, which was displaced by an anterior osteophyte. No infection occurred.

\section{Radiological Findings}

On x-ray films obtained immediately after surgery, we observed a remodeling of the bone-graft interface with progressive fading of the vertebral endplates. Thereafter, bone deposits were seen behind the graft. These deposits enlarged in time, finally coming to form a complete bone bridge between the endplates. Complete graft incorporation within bone was detected in $67(99 \%)$ of 68 operated disc levels, after a mean follow-up period of 14.9 months (range 2-59 months); (Fig. 2). In only one case, formation of a bridging bone posterior to the graft had not occurred after 22 months. This case was atypical, as the patient, in whom C6-7 fusion was performed to correct posttraumatic instability, presented with a Klippel-Feil malformation. The reduced mobility of the two upper vertebrae was probably responsible for placing increased stress on the fused segment. Despite the incomplete fusion, no spinal instability or pseudarthrosis developed.

In all cases, physiological lordosis was observed after surgery, even in patients presenting with preoperative kyphosis or spinal straightness. In eight patients in whom preoperative plain radiography studies were available, a mean gain of angulation of $11.3^{\circ}$ (range $2-26^{\circ}$ ) was measured after surgery.

Preoperatively, an additional discopathy was identified at an adjacent level in 23 patients. This lesion progressed in two cases postoperatively. In four patients complaining of moderate cervical pain a new discopathy developed after surgery.

We observed a small graft collapse $(<2 \mathrm{~mm})$ in two cases, a graft fracture without displacement in two patients, and a graft deterioration in 13 cases (Table 2). These radiological findings had no effect on the fusion or clinical outcome. No graft or plate dislocation was noted. One screw broke but remained attached to the plate by the locking device.

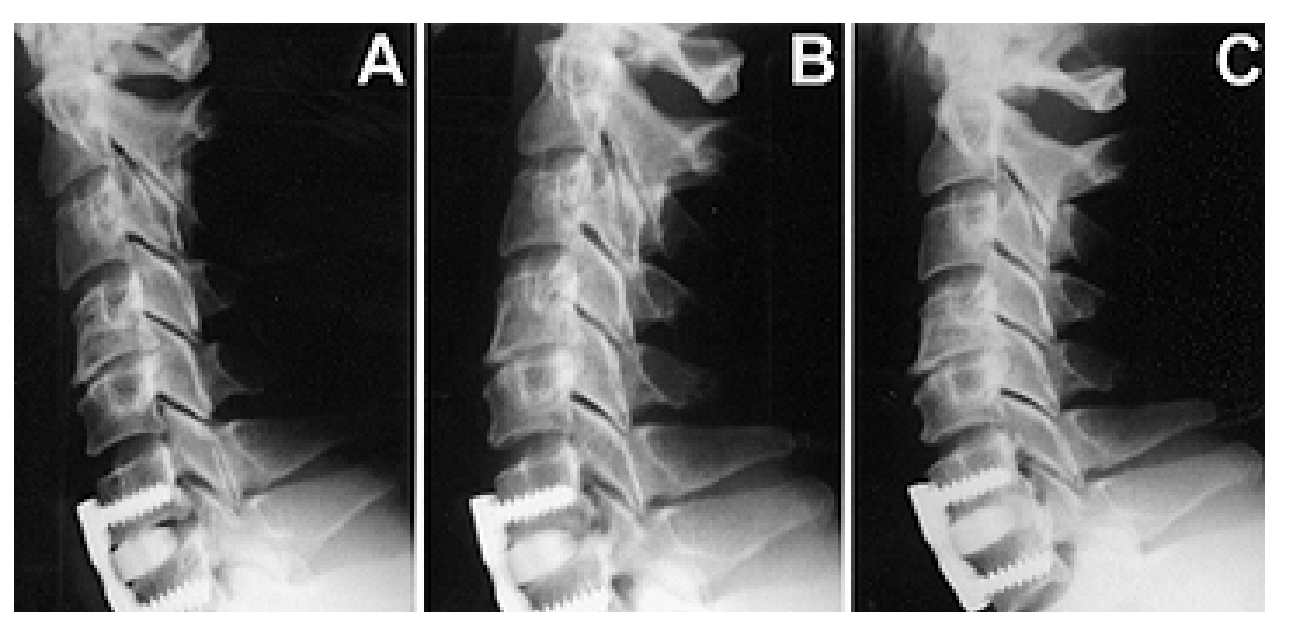

Fig. 2. Postoperative lateral cervical radiographs. A: Radiograph obtained 3 days after surgery. B: Radiograph obtained 3 months later, demonstrating remodeling of the graft-bone interface and development of a bone bridge behind the graft. C: Radiograph obtained at 12 months, revealing complete interbody fusion with graft incorporation. 
On sagittal MR imaging, artifacts induced by the plate and screws were moderate and did not prevent analysis of the fusion process. The graft and the surrounding bone were clearly visualized on $T_{1}$ - and $T_{2}$-weighted MR images (Fig. 3).

\section{DISCUSSION}

Since it was first introduced in the 1950s by Smith and Robinson, ${ }^{39}$ Cloward, ${ }^{8}$ and Dereymaeker and Mulier, ${ }^{10}$ anterior cervical interbody fusion has been extensively used with various grafts to treat segmental spondylotic myelopathy and radiculopathy. ${ }^{1-7,16-19,21,23,26,28,30,33,38,41,44,45}$ Fusion has also been advocated by numerous authors in cases of soft-disc herniation, even if its efficacy in this matter is still a source of debate. ${ }^{15,22,25,27,29,32,34-36}$ Whatever the indication, the ideal graft should not only induce a rapid and complete interbody fusion but also restore the physiological lordosis and maintain the intervertebral and foraminal height. $2,31,42$

The chemicophysical characteristics of the HA graft allow it to satisfy these goals. The HA ceramics are composed of hydroxylized calcium phosphate and are chemically identical to natural HA of bone. ${ }^{19,21}$ The process of mixing these materials leads to porous ceramics with high osteoconductive properties. The graft can be invaded by newly formed bone that grows directly into the pores. ${ }^{9,11,19,21}$ Unlike other synthetic grafts, however, there is no interposition of fibrous tissue between implant and bone. ${ }^{19}$ In experimental studies and, in particular, those in which electron microscopy observations are made, the authors have demonstrated the bioactive properties of HA grafts and their apparent ability to be directly bonded to bone, reproducing the natural bone-cementing mechanism. ${ }^{11,19}$ Unlike autografts and allografts, HA is not amenable to absorption by the host cells. Resorption of HA is very limited in both cell- and solution-mediated processes, in contrast to tricalcium phosphate compounds, which are rapidly resorbed. ${ }^{11,19,21}$

Preliminary clinical results were published in 1986 by Koyama and Handa ${ }^{23}$ and then by Senter, et al., ${ }^{38}$ in 1989 and by Böker, et al.. ${ }^{4}$ in 1993 . We also described our early experience in 1997. ${ }^{33}$ This long-term study is the first to report the use of the HA graft combined with plate placement. We have demonstrated that this graft material is very effective in inducing cervical interbody fusion. The first step of fusion was always a remodeling of the bonegraft interface. Progressively, a bone bridge appeared around the graft, mainly posteriorly, and enlarged until total incorporation of the graft occurred. The rate of complete fusion was $99 \%$, which is superior or at least comparable with that reported in the literature when other grafts were used. In only one patient, who presented with a Klippel-Feil malformation, was a partial fusion observed; however, no pseudarthrosis, spinal instability, or graft migration was noted during long-term follow-up study. Moreover, we observed fusion in all patients who underwent a two-level discectomy, although it has been suggested that a multilevel arthrodesis is often associated with a lower rate of bone union. . $^{3,716}$

We also found that the trapezoid shape of HA grafts allowed for correction of all preoperative segmental kyphot-

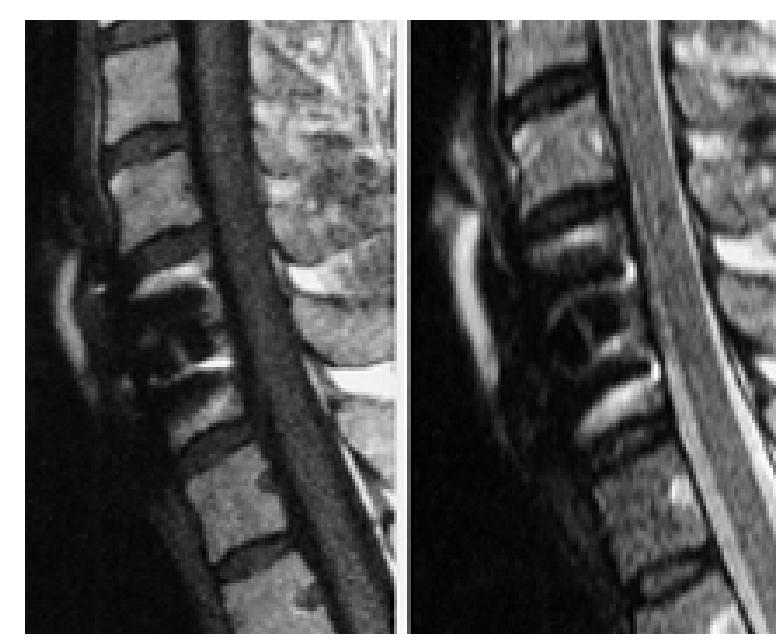

Fig. 3. Sagittal MR images obtained 1 year after surgery. Despite some artifacts produced by the plate and screws, the HA graft is clearly visible on $\mathrm{T}_{1}$ - (left) and $\mathrm{T}_{2}$-weighted images (right). Note the complete fusion of the vertebral bodies with incorporation of the graft.

ic deformities. Physiological lordosis was noted postoperatively in all patients and persisted throughout long-term review. Furthermore, intervertebral and foraminal height was maintained until fusion was complete, and no significant collapse occurred. This observation can be explained by the good resistance of the material to axial load and absence of HA resorption. The intervertebral distraction provided by the graft has several positive effects on cervical spine and decompression of the neural structures: it prevents postoperative narrowing of the foramen ${ }^{31}$ and contributes to the enlargement of the spinal canal by stretching the ligamentum flavum. ${ }^{42}$

Clinical results were quite satisfactory and comparable with those obtained in other studies in which anterior surgical procedures were conducted. ${ }^{3,5,7,15,22,25,27-29,32,34-36}$ No graft-related complication was observed. Although graft deterioration and fractures were observed radiologically, they did not impair fusion or the patient's clinical course.

Hydroxyapatite grafts, therefore, offer many advantages compared with other grafts types. Iliac crest autograft is associated with a significant donor-site morbidity and in particular pain. ${ }^{24,40}$ Moreover, the fusion rate seems lower when this graft is used. Using the Smith-Robinson technique, Bohlman, et al. ${ }^{3}$ found pseudarthrosis in 13\% of patients, $8 \%$ of whom required reoperation. Although some authors have found no relation between pseudarthrosis and fusion status, others have reported that the quality of fusion was considered to influence clinical outcome significantly, suggesting that motion at the level of the pseudarthrosis may contribute to residual nerve root compression. ${ }^{3,43}$ To improve the fusion rate, Emery, et al. ${ }^{12}$ proposed burring of the endplate. This procedure has been shown to promote fusion but at the expense of a frequent intervertebral disc space narrowing due to the autograft's settling into the vertebral cancellous bone.

The use of allografts avoids having to harvest iliac crest bone, but the authors of several studies have shown that the graft collapse occurred in 20 to $30 \%$ of cases, with a loss of intervertebral disc height up to $50 \%$. $^{1,6,45}$ The risk 
of collapse seems to be higher when cancellous bone material is used compared with cortical bone such as fibula. ${ }^{17}$ On the other hand, a significantly lower fusion rate has been reported when iliac allografts instead of autografts and fibula allografts are used. ${ }^{1,6,45}$ Moreover, in many studies in which autografts and allografts are used, the fusion rate statistically decreased as the number of fused levels increased ${ }^{3}$ as well as in patients who smoked. ${ }^{1}$ We did not observe these variations with HA grafts. Biocompatible osteoconductive polymer, ${ }^{18,26}$ methylmethacrylate $^{30,41}$ and coralline grafts ${ }^{2}$ have also been found to be poor fusion inducers. Furthermore, collapse and dislocations requiring reoperation are frequently associated with these materials. ${ }^{2}$

More recently, interbody cages have become available. These devices have been shown to produce excellent results in terms of fusion, intervertebral disc space distraction, and correction of spinal deformity. ${ }^{5,16,28}$ In two different series a $100 \%$ fusion rate was reported by authors who used carbon fiber as well as titanium cages. ${ }^{5,28}$ In one of these studies there was no case of instability, cage migration, or postoperative kyphosis. ${ }^{28}$ In most studies, however, the cages were filled with autogenous bone taken from the iliac crest $^{5}$ or at the surgical site. ${ }^{28}$

Questions may remain concerning anterior plating combined with the HA graft. In our experience, the main goal of using the plate is to ensure immediate stability and to prevent graft extrusion or fracture, by reducing the distortional forces applied to the graft during spine movements. In 1998, Kim, et al., ${ }^{21}$ described the use of an HA graft without a cervical plate in 70 patients. In three cases, anterior or posterior graft dislocation required reoperation. This complication did not occur in our study. The plate can also contribute to restoring proper spinal alignment. ${ }^{20}$ In several studies the authors have contended that correction of cervical deformities and preservation of the physiological lordosis were very important for several reasons: 1) to minimize the long-term adverse effects on the adjacent intervertebral discs; 2) to prevent delayed neurological deterioration in spondylotic myelopathy; and 3) to avoid ossification of the posterior longitudinal ligament. ${ }^{13,14,20}$ In the series reported by Kim, et al., ${ }^{21}$ without the supplemental fixation provided by the metallic plate, $40 \%$ of the patients with preoperative kyphotic deformity had segmental kyphosis postoperatively. Finally, anterior plating likely improves the fusion rate. ${ }^{37}$ During the fusion process, the graft-plate system is strengthened, thus reducing the risk of late-developing pseudarthrosis. ${ }^{37}$ Using coralline HA in a goat model, Zdeblick, et al., ${ }^{44}$ demonstrated a significant increase in the rate of graft incorporation and a decrease in the rate of collapse when a cervical plate was also implanted.

\section{CONCLUSIONS}

An HA graft is a very effective material for promoting anterior interbody fusion, whatever the indication. It allows preservation of the intervertebral space geometry, maintenance of foraminal height, and restoration of the physiological lordosis, without significant complications. The additional implantation of a cervical plate provides immediate stability, making the procedure suitable for treatment of patients who have sustained traumatic spinal injuries.

\section{References}

1. Bishop RC, Moore KA, Hadley MN: Anterior cervical interbody fusion using autogeneic and allogeneic bone graft substrate: a prospective comparative analysis. J Neurosurg 85: 206-210, 1996

2. Bizette C, Raul JS, Orhan B, et al: Résultats d'arthrodèses intersomatiques cervicales par greffons coralliens. Neurochirurgie 45:4-14, 1999

3. Bohlman HH, Emery SE, Goodfellow DB, et al: Robinson anterior cervical discectomy and arthrodesis for cervical radiculopathy. Long-term follow-up of one hundred and twenty-two patients. J Bone Joint Surg Am 75:1298-1307, 1993

4. Böker DK, Schultheiss R, van Roost D, et al: Anterior cervical discectomy and vertebral interbody fusion with hydroxy-apatite ceramic. Preliminary results. Acta Neurochir 121:191-195, 1993

5. Brooke NS, Rorke AW, King AT, et al: Preliminary experience of carbon fiber cage prostheses for treatment of cervical spine disorders. Br J Neurosurg 11:221-227, 1997

6. Brown MD, Malinin TI, Davis PB: A roentgenographic evaluation of frozen allografts versus autografts in anterior cervical spine fusions. Clin Orthop 119:231-236, 1976

7. Cauthen JC, Kinard RE, Vogler JB, et al: Outcome analysis of noninstrumented anterior cervical discectomy and interbody fusion in 348 patients. Spine 23:188-192, 1998

8. Cloward RB: The anterior approach for removal of ruptured cervical disks. J Neurosurg 15:602-617, 1958

9. Cook SD, Dalton JE, Tan EH, et al: In vivo evaluation of anterior cervical fusions with hydroxylapatite graft material. Spine 19:1856-1866, 1994

10. Dereymaeker A, Mulier J: La fusion vertébrale par voie ventrale dans la discopathie cervicale. Rev Neurol 99:597-616, 1958

11. Eggli PS, Müller W, Schenk RK: Porous hydroxyapatite and tricalcium phosphate cylinders with two different pore size ranges implanted in the cancellous bone of rabbits. A comparative histomorphometric and histologic study of bony ingrowth and implant substitution. Clin Orthop 232:127-138, 1988

12. Emery SE, Bolesta MJ, Banks MA, et al: Robinson anterior cervical fusion. Comparison of the standard and modified techniques. Spine 19:660-663, 1994

13. Goto S, Kita T: Long-term follow-up evaluation of surgery for ossification of the posterior longitudinal ligament. Spine 20: 2247-2256, 1995

14. Goto S, Mochizuki M, Kita T, et al: Anterior surgery in four consecutive technical phases for cervical spondylotic myelopathy. Spine 18: 1968-1973, 1993

15. Grisoli F, Graziani N, Fabrizi AP, et al: Anterior discectomy without fusion for treatment of cervical lateral soft disc extrusion: a follow-up of 120 cases. Neurosurgery 24:853-859, 1989

16. Hacker RJ: A randomized prospective study of an anterior cervical interbody fusion device with a minimum of 2 years of follow-up results. J Neurosurg 93 (Suppl 2):222-226, 2000

17. Hanley EN, Harvell JC, Shapiro DE, et al: Use of allograft bone in cervical spine surgery. Semin Spine Surg 1:262-270, 1989

18. Ibanez J, Carreno A, Garcia-Amorena C, et al: Results of the biocompatible osteoconductive polymer (BOP) as an intersomatic graft in anterior cervical surgery. Acta Neurochir 140: 126-133, 1998

19. Jarcho M: Calcium phosphate ceramics as hard tissue prosthetics. Clin Orthop 157:259-278, 1981

20. Katsuura A, Hukuda S, Imanaka T, et al: Anterior cervical plate used in degenerative disease can maintain cervical lordosis. J Spinal Disord 9:470-476, 1996

21. Kim P, Wakai S, Matsuo S, et al: Bisegmental cervical inter- 
body fusion using hydroxyapatite implants: surgical results and long-term observation in 70 cases. J Neurosurg 88:21-27, 1998

22. Klaiber RD, von Ammon K, Sarioglu AC: Anterior microsurgical approach for degenerative cervical disc disease. Acta Neurochir 114:36-42, 1992

23. Koyama T, Handa J: Porous hydroxyapatite ceramics for use in neurosurgical practice. Surg Neurol 25:71-73, 1986

24. Kurz LT, Garfin SR, Booth RE: Harvesting autogenous iliac bone grafts. A review of complications and techniques. Spine 14:1324-1331, 1989

25. Lunsford LD, Bissonette DJ, Jannetta PJ, et al: Anterior surgery for cervical disc disease. Part 1: treatment of lateral cervical disc herniation in 253 cases. J Neurosurg 53:1-11, 1980

26. Madawi AA, Powell M, Crockard HA: Biocompatible osteoconductive polymer versus iliac graft. A prospective comparative study for the evaluation of fusion pattern after anterior cervical discectomy. Spine 21:2123-2130, 1996

27. Martins AN: Anterior cervical discectomy with and without interbody bone graft. J Neurosurg 44:290-295, 1976

28. Matge G: Anterior interbody fusion with the BAK-cage in cervical spondylosis. Acta Neurochir 140:1-8, 1998

29. Maurice-Williams RS, Dorward NL: Extended anterior cervical discectomy without fusion: a simple and sufficient operation for most cases of cervical degenerative disease. Br J Neurosurg 10:261-266, 1996

30. McAfee PC, Bohlman HH, Ducker T, et al: Failure of stabilization of the spine with methylmethacrylate. J Bone Joint Surg Am 68:1145-1157, 1986

31. Murphy MA, Trimble MB, Piedmonte MR, et al: Changes in the cervical foraminal area after anterior discectomy with and without a graft. Neurosurgery 34:93-96, 1994

32. Pointillart V, Cernier A, Vital JM, et al: Anterior discectomy without interbody fusion for cervical disc herniation. Eur Spine J 4:45-51, 1995

33. Pollo C, De Coene B, Collard A, et al: Anterior cervical discectomy and interbody fusion with hydroxyapatite grafts and plate. Rachis 9:39-46, 1997

34. Robertson JT: Anterior removal of cervical disc without fusion. Clin Neurosurg 20:259-261, 1973
35. Rosenorn J, Hansen EB, Rosenorn MA: Anterior cervical discectomy with and without fusion. A prospective study. J Neurosurg 59:252-255, 1983

36. Savolainen S, Rinne J, Hernesniemi J: A prospective randomized study of anterior single-level cervical disc operations with long-term follow-up: surgical fusion is unnecessary. Neurosurgery 43:51-55, 1998

37. Schneeberger AG, Boos N, Schwarzenbach O, et al: Anterior cervical interbody fusion with plate fixation for chronic spondylotic radiculopathy: a 2- to 8-year follow up. J Spinal Disord 12:215-221, 1999

38. Senter HJ, Kortyna R, Kemp WR: Anterior cervical discectomy with hydroxylapatite fusion. Neurosurgery 25:39-43, 1989

39. Smith G, Robinson R: The treatment of certain cervical spine disorders by anterior removal of the intervertebral disc and interbody fusion. J Bone Joint Surg Am 40:607-624, 1958

40. Summers BN, Eisenstein SM: Donor site pain from the ilium. A complication of lumbar spine fusion. J Bone Joint Surg Br 71: 677-680, 1989

41. van den Bent MJ, Oosting J, Wouda EJ, et al: Anterior cervical discectomy with or without fusion with acrylate. A randomized trial. Spine 21:834-840, 1996

42. White AA, Panjabi MM: Clinical Biomechanics of the Spine, ed 2. Philadelphia: Lippincott, 1990

43. White AA, Southwick WO, Deponte RJ, et al: Relief of pain by anterior cervical-spine fusion for spondylosis. A report of sixtyfive patients. J Bone Joint Surg Am 55:525-534, 1973

44. Zdeblick TA, Cooke ME, Kunz DN, et al: Anterior cervical discectomy and fusion using a porous hydroxyapatite bone graft substitute. Spine 19:2348-2357, 1994

45. Zdeblick TA, Ducker TB: The use of freeze-dried allograft bone for anterior cervical fusions. Spine 16:726-729, 1991

Manuscript received February 21, 2001.

Accepted in final form March 20, 2001.

Address reprint requests to: Thierry Gustin, M.D., Department of Neurosurgery, University Hospital of Mont-Godinne, Avenue Dr. Therasse 1, B-5530 Yvoir, Belgium. email: thierry.gustin@ clin.ucl.ac.be. 